\title{
Evolving social behaviour through selection of single-cell adhesion in Dictyostelium discoideum
}

\author{
Sandrine Adiba ${ }^{1, *}$, Mathieu Forget ${ }^{1,2}$, Silvia De Monte M,2 $^{1,2}$ \\ 1 Institut de Biologie de l'ENS (IBENS), Département de biologie, Ecole normale \\ supérieure, CNRS, INSERM, Université PSL, 75005 Paris, France \\ 2 Department of Evolutionary Theory, Max Planck Institute for Evolutionary Biology, \\ Plön, Germany \\ *Corresponding author: adiba@bio.ens.psl.eu
}

\section{Abstract}

The social amoeba Dictyostelium discoideum commonly forms chimeric fruiting bodies by aggregation of different strains. Genetic variants that produce a higher proportion of spores are predicted to undercut multicellular organization unless cooperators assort positively. Cell adhesion is considered a primary factor driving such assortment, but evolution of adhesion has not been experimentally connected to changes in social performance. In this study we modified by experimental evolution the properties of individual cells, selecting for higher and lower adhesion to substrate. We then quantified the effects of these changes on cell-cell adhesion, development, and social behaviour. Unlike strains selected based on relative reproductive success in the social stage, we found that in binary chimeras both derived strains produce a smaller fraction of spores than the ancestor. Thus, evolution appears to have produced social cooperators. Examination of development revealed that this is however achieved via two opposed paths. Cells selected to be more adhesive to the substrate disproportionately contribute to the structural stability of fruiting bodies, as one would expect for cooperators. On the contrary, less adhesive cells behave as cheaters that undermine their own success more than that of the ancestor. These differences are reflected by a metric for social success that generalizes the classically used variation in frequency during the multicellular phase. Our work shows that cell mechanical interactions can constrain evolution of development and assortment in chimeras, and calls for integrating cell-level processes in conceptualizing the emergence of multicellular organization.

\section{Author summary}

The multicellular life cycle of Dictyostelium discoideum is as amazing as puzzling. Genetically different cells commonly co-aggregate, so that collective functions - that repose on cooperation among cells - are constantly threatened by the presence of exploitative variants, commonly called 'cheaters'. Most previous studies focused on how reproductive success in the multicellular phase of the life cycle can be compensated by positive assortment of cooperating cells, but recent models have stressed the impact of non-aggregated cells on the evolutionary dynamics. We use experimental evolution to explore how selection acting on single-cell adhesion impacts social behaviour. Cell adhesion is held as a fundamental mechanism for cells to direct their cooperative behaviour towards kin, but the way it interplays with social behaviour during evolution has been so far only theoretically explored. Higher levels of cooperation appear to evolve as a result of both increased and decreased cell-substrate adhesion. However, 
such phenotypes are associated to opposite developmental dynamics in chimeric aggregates. Differences in social behaviour are captured if spore production of isolated strains is also considered, suggesting that in both cases cooperative behaviour is associated to stronger adhesion.

Keywords: evolution of social behaviour; cooperation; multicellularity; experimental evolution; adhesion; Dictyostelium discoideum.

\section{Introduction}

The evolution of unicellular organisms has long been focused on properties of individual cells that live in isolation. In the recent decades, it became clear that many functions of microbial populations - spanning from stress resistance in biofilms and invasion by a pathogen to spore production in aggregative microbial species - derive from the collective, social outcome of interactions among cells [1]. Understanding how selection shapes functionality of groups of cells is challenging, in particular when multicellular aggregates are composed of multiple cellular types 22. In this case, according to a common distinction, 'cooperator' and 'cheating' lineages compete within groups and can disrupt their collective functions.

The 'social amoeba' Dictyostelium discoideum, whose life cycle comprises a multicellular body formed by aggregation of formerly independent cells, is an established model system to address the role of genetic conflicts on the evolution of multicellular organization [3,4]. Chimeric aggregates, where cells of different strains develop together and differentiate in several tissues, readily occur both in nature and in the lab 5, 6]. Within aggregates, a cell's terminal differentiation has two main issues: spores, that disperse and survive starvation, and a stalk of dead cells. The dramatic disparity in reproductive output of such two cellular fates generates strong conflicts within chimeric aggregates. 'Cheater' strains that are over-represented in the spores are expected to get enriched in successive cycles of aggregation and dispersal [3].

Assortment of cells of similar social investment is believed to be the essential factor in curtailing the evolutionary success of such 'cheaters', as it prevents them to reap collective benefits [3]. In practice, several mechanisms can underpin such assortment (e.g. spatial structure, differential adhesion, kin recognition) 7]. It is still unclear however what cell-level features implement in $D$. discoideum the general organizing principles that underpin the repeated emergence of aggregative multicellularity [8 10].

Cell adhesion has been invoked as a primary means to achieve assortment among different strains [11 17]. Beside being ubiquitous in the microbial world and essential for cell sorting in multicellular organisms, it is involved in every step of Dictyostelium life cycle. Amoebae spend most of their time in a vegetative phase, where isolated cells crawl, divide, and feed individually on bacteria [18,19]. Adhesion to their physical support determines their motility properties and changes throughout the cell cycle (mitotic cells detach from the surface) 20]. During the multicellular development, that is triggered by nutrient depletion, different forms of cell-cell adhesion come to the fore. At the beginning of aggregation, cells display non-polar cell-cell adhesion. Later, they attach head-to-tail while the streams they form converge towards aggregates, called mounds 21,22. These develop in a matrix-coated slug that displaces by collective cell motion, and finally forms a fruiting body. Despite not being independent [23], cell-substrate and cell-cell adhesion are associated to different gene expression profiles 24]. Importantly, by their implication in different stages of the life cycle, different forms of adhesion are subjected to distinct selective pressures. Selection of cell-cell adhesion presupposes a multicellular context, whose collective function will generally depend on the type and proportion of its constituent cells. Cell-substrate adhesion, on the other hand, is primarily influenced by the physical environment that 
cells experience, and is expected to evolve independently of the quality and (to a certain extent at least) quantity of other cells present in the surroundings.

With this distinction in mind, we wondered whether the social behaviour of Dictyostelium would be affected by selection acting on adhesion of single cells to a substrate. We therefore designed a directed evolution experiment where vegetative cells were selected based on their adherence to a culture vial. Contrary to trait modifications obtained by mutagenesis and KO experiments, artificial selection allows to mimic gradual phenotypic changes that are expected to occur in nature, and that may involve multiple genes simultaneously, as well as epigenetic changes. Moreover, it allows to track the evolutionary history of adaptations.

Starting from the same ancestral AX3 strain, we selected cells for increased or reduced substrate adhesion during two months of daily passages. Two derived strains, evolved under opposite selective pressure, were then characterized in terms of their phenotypes both in isolation and during development. We then considered how cell-substrate adhesion affects, directly or indirectly through cell-cell adhesion, the social behaviour of the derived strains when they co-aggregate with their common ancestor. Although the evolved strains have a recent common ancestor, their phenotypes are sufficiently divergent to significantly affect spore bias, the most common readout of social interactions. Analysing the development of chimeric fruiting bodies allowed us to identify two opposite routes by which derived strains achieve a same, apparently cooperative, behaviour. We understand these results in the light of adhesion properties of each strain in isolation, and propose an alternative metric for social performance able to reflect the observed developmental differences. We conclude discussing the potential implications of selection for substrate adhesion on the evolution of social behaviour.

\section{Materials and methods}

\section{Strains and culture condition}

Dictyostelium discoideum axenic AX3 strain (Dictybase ID: DBS0235545) was transformed with plasmids pTX-GFP (Dictybase ID: 11) or pTX-RFP (Dictybase ID: 112) to express either GFP or RFP fluorescent markers respectively. The fluorescent proteins encoded on the plasmid also carries a gene for antibiotic resistance (Gentamicin 418, Sigma-Aldrich: G418). Cells were cultured in autoclaved HL5 medium (per L, 35.5 g HL5 from formedium, $\mathrm{pH}=6.7)$ at $22^{\circ} \mathrm{C}$ and with a concentration of $20 \mu \mathrm{g} \mathrm{mL}{ }^{-1} \mathrm{G} 418$.

\section{AX3 strain transformation}

GFP and RFP-expressing cell strains were obtained by transforming cells as in 25]. Briefly, cells were transformed using a standard electroporation procedure with pTX-GFP or pTX-RFP. AX3 cells were grown in $75 \mathrm{~cm}^{2}$ flasks until they reach high density (but before they become confluent). Four to six hours before the transformation, the medium was changed. Cells were then re-suspended in $10 \mathrm{~mL}$ of ice-cold HL5 and kept on ice for 30 minutes. Cells were centrifuged for 5 minutes at $500 \mathrm{~g}$ and $4^{\circ} \mathrm{C}$. The pellet was re-suspended in $800 \mu \mathrm{L}$ of electroporation buffer and transferred into ice cold $4 \mathrm{~mm}$ electroporation cuvettes containing $30 \mathrm{\mu g}$ of plasmid DNA. Cells were electroporated at $0.85 \mathrm{kV}$ and $25 \mathrm{mF}$ twice, waiting for 5 seconds between pulses and transferred from the cuvette to $75 \mathrm{~cm}^{2}$ flask with HL5 medium. To select transformants, the next day $5 \mu \mathrm{g} \mathrm{mL} \mathrm{mL}^{-1}$ of the antibiotic $\mathrm{G} 418$ was added to the culture media. The concentration of G418 was gradually increased from $5 \mu \mathrm{g} \mathrm{mL} \mathrm{m}^{-1}$ to $20 \mu \mathrm{g} \mathrm{mL}^{-1}$ over 1-2 weeks and resistant cells were collected and frozen. 


\section{Evolution of cell-substrate adhesion}

Evolution experiments were carried out for two months by performing thirty rounds of selection by serial transfer of cells contained either in the supernatant (Top) or sticking to the culture vial after removal of the liquid (Bottom) (Fig. 11).

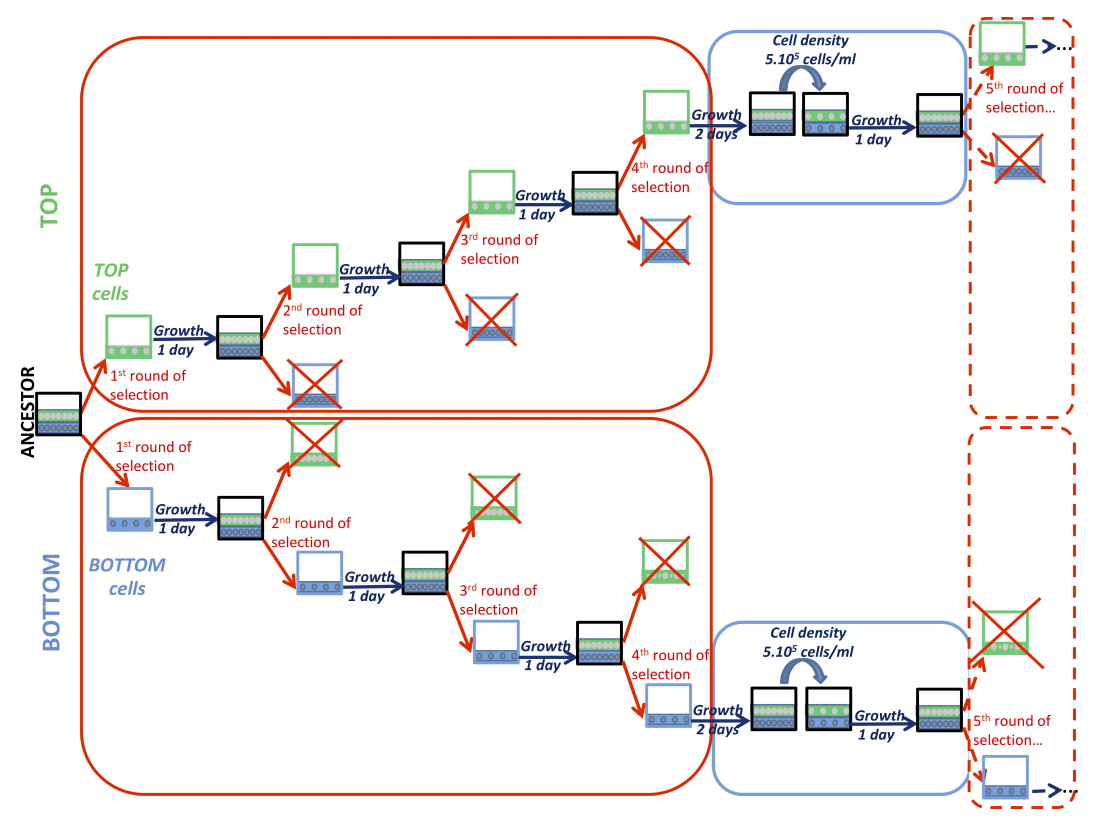

Fig 1. Evolution experiment: Artificial selection for cell-substrate adhesion phenotype: cells were subjected to two regime periods: the red box corresponds to the period of a selective pressure for cell-substrate adhesion, one round of selection per day during four days. The blue box corresponds to the growth phase (two days). The same cell density $\left(5.10^{5}\right.$ cells $\left./ \mathrm{ml}\right)$ was then obtained by dilution for all replicates and treatments before starting again the selection regime. Top lines were obtained by serial transfer of cells in the supernatant. Bottom lines were obtained by serial transfer of cells attached to the bottom flask.

To avoid that the non-adhering fraction got enriched in multi-nucleated cells (similar to what happens when cells are grown in agitation), four days of selection were alternated with two days of growth without selection. After this growth period, cell density was adjusted to $5.10^{5}$ cells.mL ${ }^{-1}$ in $10 \mathrm{~mL}$ HL5 with $20 \mathrm{ug} \mathrm{mL}^{-1} \mathrm{G} 418$ in 25 $\mathrm{cm}^{2}$ flasks and the selection regime started again the next day. During the four days of selection, the cell culture was transferred each day as follows: (i) In Top strains, the culture flask was lightly shaken to detach cells which were not strongly attached to the bottom surface and to homogenize. A volume of $5 \mathrm{~mL}$ of the liquid was then transferred in $5 \mathrm{ml}$ HL5 with $20 \mathrm{\mu g} \mathrm{mL} \mathrm{m}^{-1} \mathrm{G} 418$ in $25 \mathrm{~cm}^{2}$ flasks. (ii) Bottom strains were obtained from the cells that remained attached to the culture flask after lightly shaking the culture flask and removing the liquid. A volume of $10 \mathrm{~mL}$ HL5 with $20 \mu \mathrm{gL}^{-1} \mathrm{G} 418$ was added to wash the flask. Cells were detached by 10 to 15 cycles of pipetting with a Pasteur Pipette. A volume of $5 \mathrm{~mL}$ of the resulting cell suspension was then transferred

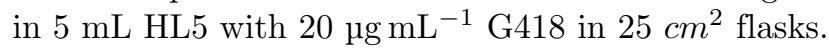

The thirty rounds of selection correspond roughly to 85 and 97 generations (estimated in S1 Fig) for the Top and Bottom strain, respectively. During this experiment, cells were kept in vegetative growth, so that selection only acted on single-cell, and not on multicellular traits. 


\section{Developmental life cycle}

Cells were grown in $25 \mathrm{~cm}^{2}$ flasks with $10 \mathrm{~mL}$ HL5 medium with $20 \mu \mathrm{g} \mathrm{mL}{ }^{-1} \mathrm{G} 418$ until they reached the mid-exponential growth phase. Cell density at the onset of starvation was comparable for all starvation assays. Cells were washed from the nutrient medium by three successive centrifugations with SorC buffer (per L, $0.0555 \mathrm{~g} \mathrm{CaCl}_{2}$; $0.55 \mathrm{~g} \mathrm{Na}_{2} \mathrm{HPO}_{4}, 7 \mathrm{H}_{2} \mathrm{O} ; 2 \mathrm{~g} \mathrm{K \textrm {K } _ { 2 }} \mathrm{PO}_{4}$ ) at $500 \mathrm{~g}$ for $7 \mathrm{~min}$. After the last centrifugation, the pellet was re-suspended in SorC buffer and the concentration adjusted to $2.10^{7}$ cells. $\mathrm{mL}^{-1}$. A volume of $40 \mu \mathrm{L}$ (corresponding to $4.10^{6}$ cells $/ \mathrm{cm}^{2}$ ) of suspension was plated on $6 \mathrm{~cm}$ plates filled with $2 \mathrm{~mL}$ of $2 \%$ Phytagel (Sigma-Aldrich) as previously described by Dubravcic et al. 26. We produced chimeras by mixing equal amounts of each evolved strain (marked with GFP) with the ancestor strain (marked with RFP).

The concentration of each strain was adjusted to $2.10^{7}$ cells. $\mathrm{mL}^{-1}$ and a volume of 20 $\mathrm{\mu L}$ of each strain mixed before plating (corresponding to a total of $4.10^{6}$ cells $/ \mathrm{cm}^{2}$ plated). The developmental cycle was subsequently imaged as explained in paragraph 'Time-lapse microscopy and image analysis'.

\section{Cell-substrate adhesion assay}

Efficiency of cell-substrate adhesion was estimated by measuring the fraction of cells attached to a culture flask. Be $N$ the total number of cells in a culture and $n_{L}$ those that are suspended in the liquid, this is $\left(N-n_{L}\right) / N$. These quantities were measured (see protocol below) for the Top, Bottom and Ancestor strains in three different conditions: 1 ) at the beginning of exponential growth (24 hours after initiating a culture at $5.10^{5}$ cells. $\left.\mathrm{mL}^{-1}\right) ; 2$ ) right after starvation, where cells at the beginning of exponential growth were washed out of the nutrient medium by three successive centrifugations with SorC buffer at $500 \mathrm{~g}$ for $7 \mathrm{~min} ; 3)$ during streaming, where cells were collected at the streaming stage of the developmental cycle (whose timing was identified by analysing time-lapse movies) by washing plates with $1 \mathrm{~mL}$ SorC to disaggregate clusters. Cultures in these three conditions were resuspended to a density of $5.10^{5}$ cells. $\mathrm{mL}^{-1}$ in $10 \mathrm{~mL}$ medium (HL5 with $20 \mu \mathrm{g} \mathrm{mL}{ }^{-1} \mathrm{G} 418$ for condition 1 and SorC for conditions 2 and 3 ) in $25 \mathrm{~cm}^{2}$ flasks. This concentration was low enough so that cells could independently attach to the surface. The total cell number $N$ was then counted using a hemocytometer. Cultures were let rest for 30 minutes, after which flasks were lightly shaken to detach cells that were not strongly attached to the bottom. The supernatant was collected into $15 \mathrm{~mL}$ tube and the number of cells in suspension $n_{L}$ measured with a hemocytometer. All measures have been realized in triplicates, starting from the same culture.

\section{Cell-cell adhesion assay}

Cell-cell adhesion was quantified by the method of Gerish 27. The principle of this method is to allow cells to get in contact with each other in a shaken culture, and then measure the proportion of cells that are found in clusters. The percentage of clustered cells was determined by subtraction of the total cell number and the number of unaggregated cells divided by the total cell number. The cell culture was centrifuged and resuspended at a density of $2.10^{6}$ cells. $\mathrm{mL}^{-1}$ in $2 \mathrm{~mL}$ of SorC buffer. This cell density was chosen so that only a fraction of cells aggregate. In the EDTA assays, 10 $\mathrm{mM}$ EDTA were added. The culture was rotated at $150 \mathrm{rpm}$ and $22^{\circ} \mathrm{C}$ during 45 minutes. The total number of cells and number of unaggregated cells were subsequently measured using a hemocytometer. The counting protocol was repeated for cultures prepared in conditions $2-3$ as described in 'Cell-substrate adhesion assay', and in triplicate for each of the Top, Bottom and Ancestor strains. 


\section{Time-lapse microscopy and image analysis}

Cells were starved as described in section 'Developmental life cycle'. The $6 \mathrm{~cm}$ diameter Petri dish was imaged on an automated inverted microscope Zeiss Axio Observer Z1 with a Camera Orca Flash 4.0 LT Hamamatsu and using a 5X objective. Images were acquired with MicroManager 1.4 software. This setup allows Petri dish scanning at regular time intervals (typically $5 \mathrm{~min}$ ), with phase contrast and fluorescence image acquisition at all time points (with $33 \mathrm{~ms}$ exposure times). A mosaic image is reconstructed by combining all the images of contiguous areas of the Petri dish at a given time point using a custom Python program. Time-lapse movies were captured in duplicates for clonal (Top, Bottom, Ancestor) and chimeric development (Top-Ancestor, Bottom-Ancestor, Ancestor GFP - Ancestor RFP).

Timing of development. By visually inspecting the time-lapse movies for Top, Bottom and Ancestor strains and their chimeras, six characteristic developmental stages were identified : streaming, mound, tipped aggregate, slug, mexican hat and fruiting body. The moment at which each developmental stage appeared for the first time was used to partition the developmental cycle.

Aggregate size and number. Aggregates size and number change during the aggregation process. In order to compare different movies, a custom imageJ macro 28 was written to compute them at the time point when their values stabilize, indicating the end of the aggregation process. At this point, aggregates area was averaged over the whole aggregation domain.

Cell position in binary chimeras during developmental life cycle. Time lapse movies were realized as previously described also for 1:1 binary chimeras where GFP-labelled derived strains, and the GFP-labelled Ancestor for the control, were mixed with RFP-labelled Ancestor cells.

\section{Measures of spore formation efficiency and metrics of social performance}

Evolved and Ancestor strains were subjected to the developmental life cycle either clonally or in binary chimeras (as described in section 'Developmental life cycle'). In addition, ancestral RFP and ancestral GFP were mixed to control for the possible differential effects of different plasmid insertion. Each experiment (clonal ancestor and derived strains, and their chimeras with the ancestor) was realised in three replicates.

The initial number of cells plated was estimated using a hemocytometer. At the end of the developmental life cycle, spores were collected using $1 \mathrm{~mL}$ pipette tips after washing the plates with $1 \mathrm{~mL}$ SorC. The number of spores was counted using the hemocytometer. Spore Formation Efficiency $S F E_{i}$ of a strain $i \in\{$ Top, Bottom, Ancestor $\}$ was measured as the ratio between the number $N_{i}$ of spores produced by that strain and the number of cells of the same strain plated at the beginning of the experiment $n_{i}$ :

$$
S F E_{i}=\frac{N_{i}}{n_{i}}
$$

Here, lower and upper case indicate the composition at the beginning $(n)$ and at the end $(N)$ of multicellular development, when is established the spore pool that will seed the following generation.

In chimeras, the proportion of cells of the two types was quantified using a flow cytometer Cube8 Partec with FL1 (GFP), FL3 ( RFP), SSC (Side Scatter) and FSC (Forward Scatter) channels. The proportion in the plated population was assessed by diluting $10 \mu \mathrm{L}$ of the initial suspension in $990 \mu \mathrm{L}$ of SorC. The proportion in the spores was similarly measured. Since the cytometer does not clearly differentiate between spores and non aggregated cells, the latter were eliminated by adding first $0.05 \%$ SDS 
for 5 minutes followed by a centrifugation at $250 \mathrm{~g}$ for 3 minutes. The pellet was re-suspended in $1 \mathrm{~mL}$ SorC before passage in the cytometer.

For binary mixtures with strain $j$, social performance of strain $i$ was quantified in two ways, starting from the measure of the fraction of spores $P_{i}$ :

$$
P_{i}=\frac{N_{i}}{N_{i}+N_{j}}
$$

and the fraction $p_{i}$ of plated cells of strain $i$ :

$$
p_{i}=\frac{n_{i}}{n_{i}+n_{j}},
$$

that was close to $50 \%$. First, following classical estimation of social behavior [29 32], we simply assessed the variation in strain's frequency during multicellular development:

$$
\text { SporeBias }_{i}=P_{i}-p_{i}
$$

This metrics is commonly used as a measure of relative fitness of strain $i$, as it quantifies the difference between the probability that a cell of type $i$ becomes a spore with respect to the frequency of that type in the population. However, as noticed by Buttery et al. [33], this formula is a good estimator of reproductive success only as long as strains produce in isolation a comparable amount of spores. Indeed, if strains can be found in clonal as well as chimeric groups, the social performance should also take into account the spore production success of strains in isolation.

In order to take into account differences in spore production between strains, eq. 2 can be generalized by estimating the number of spores of type $i$ that are expected, based on each strain's SFE in isolation (eq. 1), when there is no interaction among the strains. This quantity, that now depends on the behaviour of both strains in clonal development, is:

$$
\tilde{P}_{i}^{j}=\frac{p_{i} S F E_{i}}{p_{i} S F E_{i}+\left(1-p_{i}\right) S F E_{j}},
$$

where the expected proportion of spores depends on the initial frequency of the two strains.

Social performance of strain $i$ in a chimera with strain $j$ is then defined as the deviation of the observed proportion of $i$ spores with respect to its expected value when the two strains do not interact:

$$
d_{i}^{j}=P_{i}-\tilde{P}_{i}^{j}
$$

Notice that if the two strains have the same spore formation efficiency, then $\tilde{P}_{i}^{j}=p_{i}$ and $d_{i}^{j}=$ SporeBias $_{i}$. Moreover, it is easy to see that in binary mixes $\tilde{P}_{i}^{j}=\tilde{P}_{j}^{i}$, so that this metric respects the symmetry expected for relative fitness advantages.

For both metrics, a negative value indicates a decreasing contribution to the spore pool and is considered as indication of a strain's cooperative behaviour. On the opposite, a positive value indicates a higher contribution to the spore pool than expected, which is indicative of social cheating.

\section{Statistical analysis}

Statistical analysis was performed in R Studio. Significant difference between the samples was calculated using two-sample $t$ test function, and a $p$-value $p<0.05$ was considered as significant. 


\section{Results}

D. discoideum cells were subjected to a directed evolution experiment, where selection was applied to their adhesion to a substrate (see Methods: Evolution of cell-substrate adhesion). Serial passages to fresh medium were realized by separating cells that remained in suspension from cells attached to the bottom of the culture flask. Nutrients availability was sufficiently large to keep cells in the exponential phase of growth, thus in their vegetative unicellular state. After 30 rounds of selection (corresponding to 85-97 generations, see S1 Fig, we disposed of two derived strains, that in the following we refer to as Top and Bottom respectively. We expect these derived strains to harbour genetic or epigenetic variations that underpin differences in adhesion with respect to the Ancestral Ax3 strain. This was confirmed by the observation that phenotypes were reproducible even after multiple cycles of growth and dilution. In order to exclude the effect of non-heritable differences in physiological state, all the experiments that follow were realized after frozen stocks were thawed and grown before adjusting the same cell density. Population-level assays described in the following were realized in three replicates started from the same initial culture, while time-lapse movies were captured in duplicates.

As expected, at the end of the evolution experiment, Top, Bottom and Ancestral cells differed in their adhesion to the substrate, assessed in early exponential growth phase (see Methods: Cell-substrate adhesion assay). Top cells were twice less adhesive than the Ancestor, while Bottom ones displayed a 20\% increase in adhesion (S2 Fig).

\section{Differential cell-substrate adhesion affects multicellular development}

The multicellular stage of the life cycle was not directly subjected to selection. In the absence of pleiotropic effects of cell-substrate adhesion, its traits should therefore be chiefly affected by drift, thus they may not undergo directional evolutionary variation. In this section, we investigate the developmental cycle of the derived strains and show that it differs with respect to their common ancestor. We also explore how the observed deviations relate to differences in cell adhesion properties.

As a first step, we observed clonal multicellular development for the three different strains, starting from the same initial cell density (see Methods: Developmental life cycle). Derived strains are still able to perform the developmental life cycle from aggregation to the formation of the fruiting bodies S3 Movie: Ancestor, Top and Bottom alone). However, the timing of each stage (aggregation, streaming, mound, tipped aggregate, slug and mexican hat) varies considerably and reproducibly among strains (Fig. 2). 


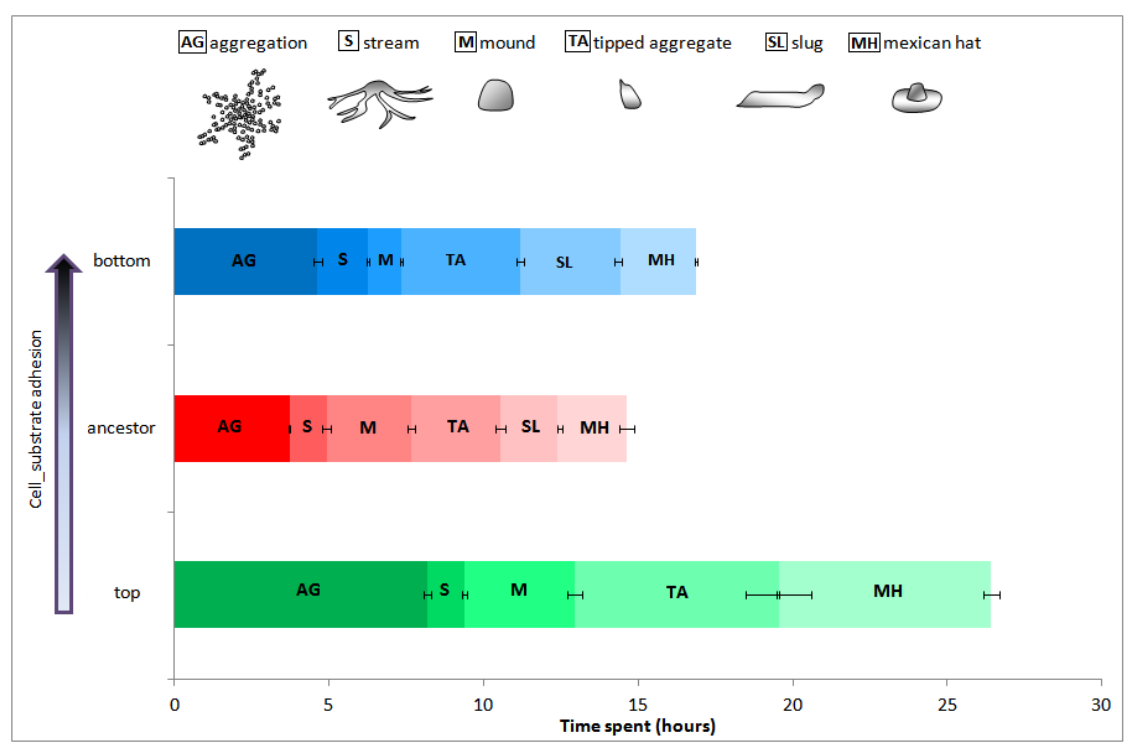

Fig 2. Timing of developmental life cycle.

The different stages of the developmental cycle were classified by analysing phase contrast time lapse movies (see Methods: Time-lapse microscopy and image analysis). The figure displays the mean of two independent experiments for Top (green), Ancestor (red) and Bottom (blue) cells. Differences in development, most notable for the Top strain, are discussed in the text.

Figure 3 shows snapshots of such different stages in the Ancestor, Top and Bottom strains.

The developmental cycle of Bottom cells, that were more adherent to the substrate in exponential phase, is similar to that of the Ancestor strain. Five hours after plating, the firsts streams are observed. For the same initial cell density, the Bottom strain tends to form a smaller number of aggregates (S4 Fig B). Mounds have, on average, a smaller diameter (S4 Fig A). This does not necessarily mean they contain less cells, as differences in the area covered by the aggregate may be due to the influence of cell-substrate adhesion on the shape of the mound. Correspondingly, slugs of the Bottom strain migrate longer and fruiting bodies are noticeably taller, indicating a higher propensity of Bottom cells to differentiate in the stalk. The Top strain, on the other hand, is more severely impaired in its development (Fig 3 ).

From the beginning, Top cells tend to float and readily form small clusters S4 Fig A). Streaming starts 7.5 hours after plating, much later than for the Ancestor. Similarly, the first stages of multicellular development are considerably delayed. Streams tend to break, mounds are more numerous and of smaller area (S4 FigA-B). The most notable qualitative difference with respect to the other strains is that there is no slug stage, and tipped mounds directly develop into small fruiting bodies, leaving off numerous cells in proximity of the basal disc. Moreover, formation of the fruiting body often fails, suggesting that reduced cell-substrate adhesion plays a key role in the final stages of multicellular development. Particularly, it appears to impair the organization of the tip, which defines the developmental axis of the slug in the Ancestor [34], and the anchoring of the sorocarp onto the surface. 


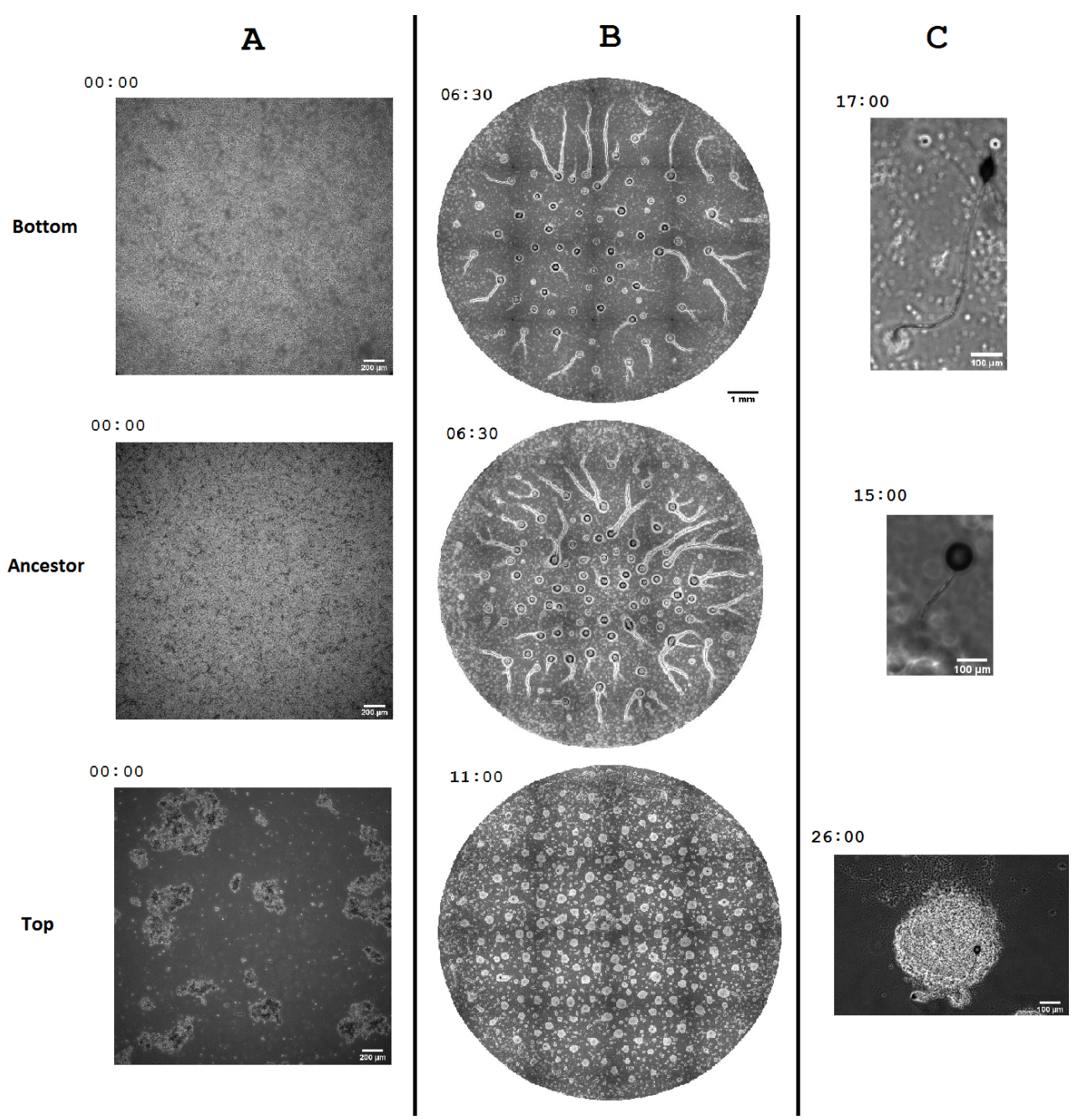

Fig 3. Developmental cycle of the Top, Ancestor and Bottom strains.

Snapshots are taken at equivalent developmental phases, rather than at a same absolute time, in order to meaningfully compare the different strains (see Methods: Time-lapse microscopy and image analysis). Phase contrast images of three stages of the cycle: beginning of aggregation (A), mounds (B), fruiting bodies (C). The time of capture is indicated on the left of each picture (see Fig. 2 for reference). As discussed in the text, strains selected for their adhesion to the surface differ significantly from the Ancestor also in their developmental cycle, with the Top strain displaying the largest variations.

Altered developmental patterns in the derived strains resulted in a lower number of spores produced (Fig. 4).

The observed differences can be attributed either - for Bottom cells - to the formation of fruiting bodies with a disproportionate fraction of stalk cells or - for Top cells - to frequent failure to complete multicellular development, which we expect to be at least partially rescued in chimeras. Cell-substrate adhesion thus appears to affect non-monotonously reproductive output. 


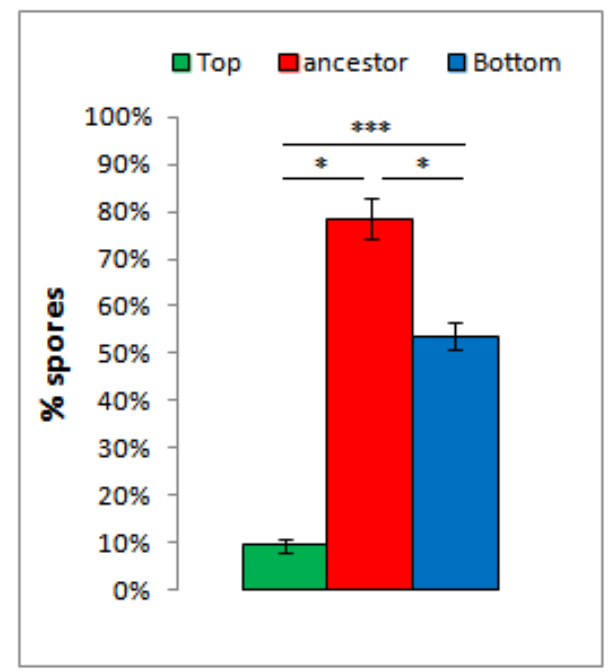

Fig 4. Spore formation efficiency in derived and Ancestral strains. As a result of impaired development, both Top and Bottom derived strains produce fewer spores than the Ancestor $\left({ }^{*} \mathrm{p}<0.05,{ }^{* *} \mathrm{p}<0.001,{ }^{* * *} \mathrm{p}<0.0001\right.$, colour code as in Fig. 2p.

\section{Differences in cell-substrate adhesion affect cell-cell adhesion during development}

Modifications of cell-substrate adhesion in the selected strains can influence multicellular development either directly, or indirectly through some co-varying trait that has direct developmental effect. In the former case at least, adhesion differences should be maintained to some extent during the developmental cycle. We therefore assess, first, cell-substrate adhesion outside the vegetative growth phase of the life cycle, when selection was applied. Later, we will turn to consider another kind of adhesion that among cells - along the developmental cycle.

On top of the previous observations realized in exponential growth (S2 Fig), we measured cell-substrate adhesion at two additional time points. 'Starvation' is just after cells were transferred into buffer, before being plated to start the developmental cycle, and is the same for all strains. 'Streaming', marked by the onset of the streaming phase, corresponds instead to different times after plating, depending on the strain (five hours for Ancestor and Bottom cells and seven hours and a half for Top cells). For the latter time point, measures were realized on cells collected from a plate, where they had started their developmental cycle.

Cell-substrate adhesion differences remain qualitatively the same at different time points: Top cells remain the less adhesive and Bottom cells the most adhesive to the substrate. In accordance with previous studies 24, cell adhesion is however modulated along the developmental life cycle. With respect to exponential phase in rich medium, cell-substrate adhesion is increased after starvation in all strains, and decreases (in a more pronounced way in Top cells) at the streaming stage.

Persistent differences in cell-substrate adhesion may be sufficient to explain why strains have distinct developmental trajectories. On the other hand, during multicellular development the quintessential form of adhesion is that among cells. As these two forms of adhesion have been documented to co-vary [23], we consider cell-cell adhesion as a natural candidate for exploring pleiotropic effects of selected variants in cell-substrate adhesion.

The protocol by Gerisch 27] (Methods: Cell-cell adhesion assay) allowed us to quantify the capacity of single cells in suspension - thus independent of their substrate 
adhesion - to form clusters. We observed that cell-cell adhesion was consistently lower in the Ancestor than in the derived strains at starvation and streaming time points (Fig 5 .

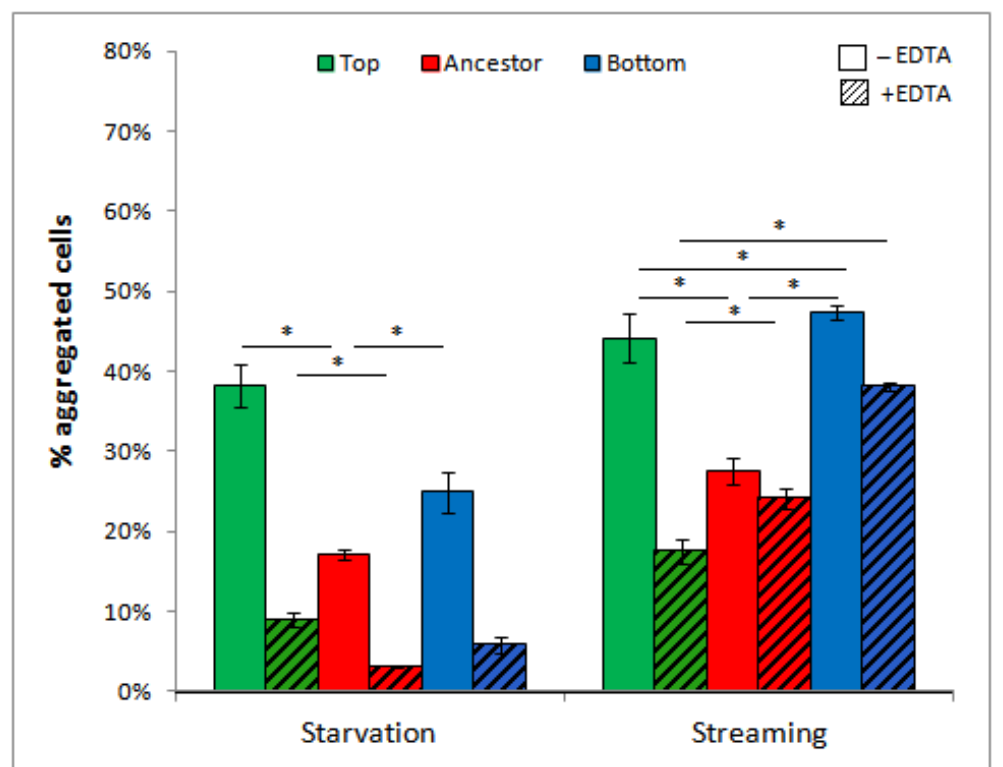

Fig 5. Cell-cell adhesion differences through development.

The percentage of aggregated cells at the beginning of starvation and during streaming is higher for the derived strains than the Ancestor. Differential decrease of such percentage upon addition of EDTA (patterned bars) suggests that higher cell-cell adhesion of Top cells is mostly due to early-development adhesion proteins $\left({ }^{*} \mathrm{p}<0.05\right.$, ${ }^{* *} \mathrm{p}<0.001$, colour code as in Fig. 2.

We tried to get further insights on the origin of such differences by reasoning that changes in cell-cell adhesion are unlikely to involve newly evolved contact sites, and that selection has probably acted on existing adhesion systems. Two families of proteins are known to be involved in cell-cell adhesion in Dictyostelium. The adhesion system DdCAD-1 (gp24) is expressed soon after starvation and is inhibited by Ethylene Diamine Tetra acetic Acid (EDTA) 35, 36. Later, in the streaming and mound phases, polar cell adhesion by EDTA-resistant gp80 glycoproteins causes cells to organize in chains 37 and elicits activation of the cAMP-mediated signal transduction pathway 38]. Addition of EDTA to the culture, together with the observation in two different developmental phases, informed us on the part that mechanisms involved in the early stages of multicellular development have on increased cell-cell adhesion of derived strains.

At starvation, EDTA drastically reduced cell clustering, as expected. It is interesting however to notice that this reduction happens in similar proportions for all strains, suggesting that also in normal conditions surface adhesion does not alter massively the probability of cell encounter, but rather that of cells sticking to one another. During streaming, EDTA produces a small decrease in clustering for Ancestor and Botttom strains, that seem thus to maintain their polar adhesion intact (but stronger for the Bottom strain). Instead, it is highly disruptive for the Top strain, suggesting that increased cell-cell adhesion relies on different mechanisms for Top and Bottom cells: adhesion proteins typical of early development, and proteins responsible for polar contacts, respectively. This also explains why Top cells are more adhesive than Bottom earlier, while the reverse is true later. Moreover, it is consistent with the previous 

variation in cell-cell adhesion might be due to a lower expression level of the gp 80 adhesion protein.

Strains selected for altered cell-substrate adhesion in the unicellular phase of the life cycle thus display also differences in cell-cell adhesion. Such differences correlate to modifications of developmental patterns, thus with their social behaviour in genetically homogeneous multicellular aggregates. We can now wonder whether they also affect social behaviour in chimeras.

\section{Developmental patterns of derived strains in chimeras}

\section{Social success and spore formation efficiency}

We mixed a reference Ancestor strain (marked with RFP) to the two derived strains and the Ancestor, all marked with GFP. In the following, we refer to chimeras where Ancestor cells were mixed to an equal amount of Bottom (Top, Ancestor) cells, while maintaining the same total cell number as for clonal development, as BA (TA,AA, respectively). We quantified the composition of the spore pool by counting the total number of spores and measuring the fraction of spores belonging to each strain (see Methods: Measures of spore formation efficiency and metrics of social performance). First, we used the AA mix to check that differences in fluorescent labelling plasmid did not per se induce a spore bias (see S5 Fig).

Classically, social behaviour in chimeras is assessed by measuring the proportion of spores produced by each of the two strains. This is compared to the composition of the initial mix (typically 50\% of cells of each strain, Equation 2 in Methods: Measures of spore formation efficiency and metrics of social performance) 29,32 in order to determine how much the proportion of one type is enriched in the spores. If the two strains differ in the number of spores they produce, however, it has been argued that the expected proportion will depend on their clonal spore formation efficiency 33]. Equation 4 in Methods: Measures of spore formation efficiency and metrics of social performance generalizes the previous metric to this case. The expected number of spores is now calculated assuming that each strain contributes the same fraction of spores in isolation and in chimeras, hence that the decision to differentiate into a spore is independent of the other strain.

When mixed with the ancestor strain, both derived strains contribute to the spore pool less that their initial proportion in the mix (S5 Fig A). Based on the widely used social metric Equation 2, they behave as 'cooperators' towards the Ancestor, because they are under-represented in the spore pool, thus in a following generation, compared to the latter. If they appear to help the ancestor by allowing its cells to be over-represented in the spores, however, they also cause its spore production to diminish S5 Fig B). When the clonal spore formation efficiency of each strain is taken into account to estimate the deviation through Equation 4, it becomes evident that Top and Bottom cells differ in their social strategy. The Top strain behaves as a cheater, as in its presence Ancestor cells cooperate, forming less spores than expected. Bottom cells, on the opposite, behave as cooperators as their spore production is lower than expected. The results of pair-wise comparison of spore production of different strains in isolation and in chimeras are collected in S6 Table: Metrics of reproductive success in chimeras and deviations in competitive spore allocation of each strain compared to their inherent allocation,

Despite qualitatively similar reductions in the percentage of spores produced and in cell-cell adhesion properties, selected variations of cell-substrate adhesion in Top and 
Bottom strains thus appear to have opposite effects on social behaviour. Understanding how selection in the unicellular phase of the life cycle affects social interactions and how derived strains end up being under-represented in the spore pool requires examining cell behaviour in the course of the developmental cycle.

\section{Differential adhesion affects cell aggregation and sorting during streaming and within slugs}

Derived strains are marked with GFP. This allows us to observe how cells of different strains behave during development in mixes with the Ancestor strain, that we marked with a RFP.

For all binary mixes, cells were deposed on phytagel so that labelled cells were uniformly randomly distributed at the beginning of development. The developmental cycle was then followed by time-lapse microscopy. We first checked that the different fluorescent markers did not give rise to segregation during aggregation or development (Fig. 7 middle; S7 Movie: multicellular developmental cycle in a chimera between Ancestor GFP and RFP). Consistent with the observed lack of bias in the spores, no distinctive pattern was recognizable at any developmental stage and the developmental timing was comparable to that of single Ancestor populations (Fig. 6).

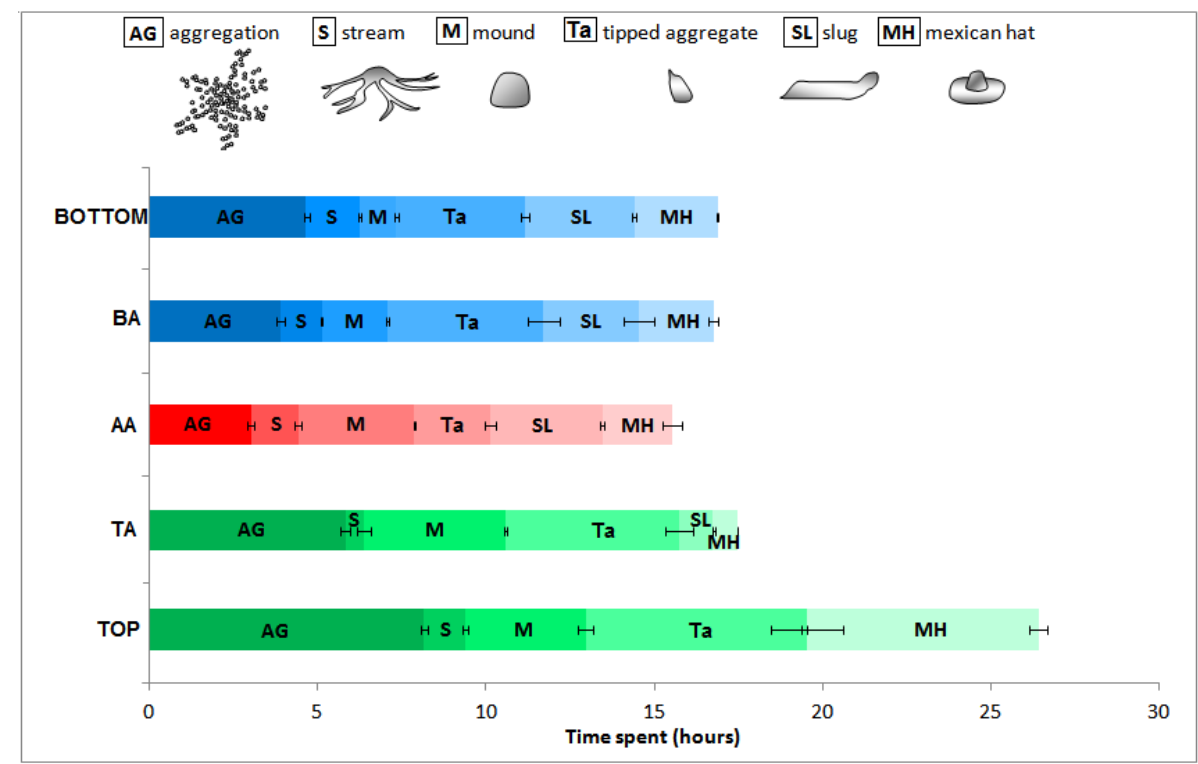

Fig 6. Timing of the developmental life cycle in chimeras.

Chimeras of Bottom-Ancestor (BA); Ancestor GFP -Ancestor RFP (AA) and Top-Ancestor (TA) are compared in the timing of the developmental cycle to single-strain multicellular cycle. AA behaves like the pure Ancestor. Each phase starts with the first apparition of the different multicellular stages, indicated on top in time-lapse movies S8 Movie: multicellular developmental cycle in a chimera between Top and Ancestor, S9 Movie: multicellular developmental cycle in a chimera between Bottom and Ancestor). Colour code as in Fig. 2

Anomalies in developmental timing observed in pure Bottom and Top cultures were largely rescued by mixing with the Ancestor (Fig. 6), as often happens with mutants deficient in some multicellular function. Most notably, Top cells, that do not form slugs on their own, recovered the normal succession of developmental phases, though their development was still slightly slowed down. 
In order to compare the behaviour of Top and Bottom strains in chimeras with the Ancestor, in the following we describe how the two types behave in successive stages of the developmental cycle. Looking at the position of cells, we establish connections with previously characterized differences - among strains and during developmental time - in cell-substrate and cell-cell adhesion, and elucidate the mechanistic bases of the observed social behaviour (Fig. 7).

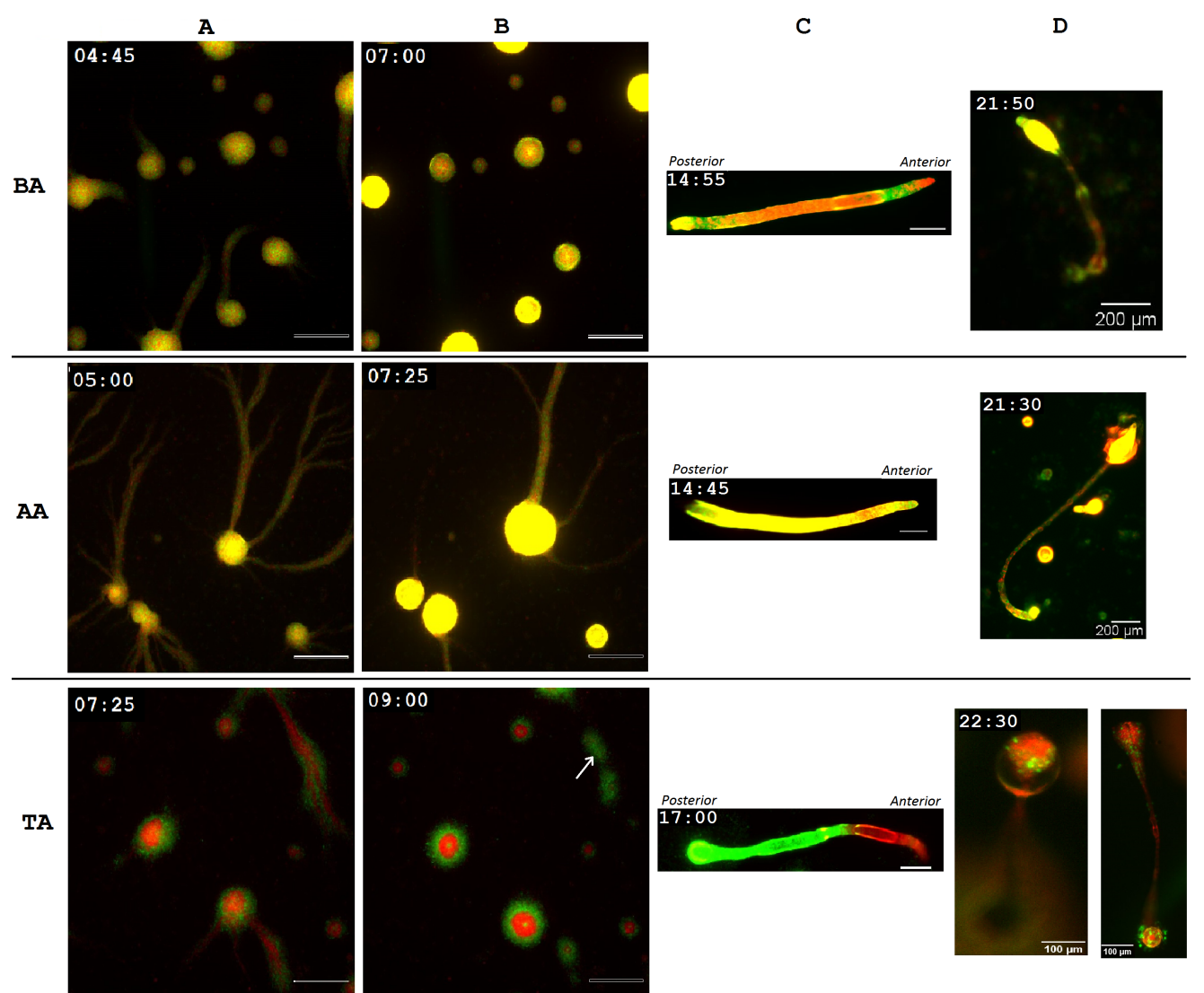

Fig 7. Developmental patterns in chimeras. Snapshots from time-lapse movies during multicellular development in chimeras BA (top), AA (middle) and TA (bottom). Ancestor strain is marked with RFP (and GFP for control) and evolved strains with GFP. Cultures with an equal proportion of each strain were starved and plated at the same total density (Methods 'Developmental life cycle'). (A) In Early aggregation, Top cells are positioned at the edge of the streams and at the periphery of aggregates (Scale bar $=500 \mu \mathrm{m})$. (B) Cells that enter the mound last (the arrow indicates a stream in the late aggregation stage) are predominantly Top cells. In the mound, Top cells are found in the center and periphery of the aggregate (Scale bar $=500 \mu \mathrm{m})$, producing a bull's eye pattern. (C) In the slug, Bottom cells are mainly localized in regions that later develop into upper cup and basal disk, while Top cells are enriched at the back of the slug. Scale bar $=200 \mu \mathrm{m}$. (D) In the fruiting bodies, Bottom cells are concentrated in the tip, the upper cup, and the basal disk, and Top cells are prominently found in the spore ball, and in the basal disc.

BA chimeras display no bias in cell localization before the slug stage. In TA mixes, instead, Top cells are found predominantly on the exterior part of streams. As they attach less to the surface, Top cells appear to be dragged passively in the streams and within the mound by Ancestor cells. Even if some Top cells appear to join aggregates 
concomitantly with Ancestor cells, streams that reach aggregates latest are almost exclusively composed of Top cells. This physical sorting continues in the mound phase. During development of the Ancestor, cells - previously organized in head-tail chains inside the streams - turn around the aggregate center, maintaining their polar orientations. Top cells, that have low polar adhesion, get excluded from the rotating ring and accumulate at the outer boundary of the mound and in its center. This gives to the mound a bull-eye appearance (Fig. 7 B (TA)).

Bottom and Ancestor cells appear interspersed in BA chimeric mounds, reflecting the capacity of both strains to establish polar contacts (though with different intensity). However, differences in substrate adhesion and, possibly, in cell-cell adhesion affect cell sorting within slugs. Bottom cells tend to be enriched in the pre-stalk, anterior region of the slug (but not on the very tip) and in the posterior zone (but not the very rear). Similar to pstAO and pstAB cells, that compose the back part of the slug head in normal development, they appear to migrate towards the rear of the slug and eventually contribute disproportionately to the basal disc and the upper cup. Both these positions require strong adhesion: the basal disk keeps the fruiting body attached to the surface, and the upper cup 'pushes' the spore mass along the stalk. Bottom cells thus behave as expected for 'cooperators', as their adhesion properties make them take on roles that are fundamental for the structural stability and function of the sorocarp.

In TA chimeras, the very distinctive bull-eye pattern of the mound reflects in the later geometry of the slug. From the tipped aggregate stage, it is the Ancestor cells that drive the formation of the tip and the emergence of the slug, whereas Top cells tend to remain to the margins. Even if they have weaker polar attachment, they manage nonetheless to be carried along by Ancestor cells, whose intact cell-substrate adhesion propels the collective motion of the multicellular stage. Top cells are thus eventually found in the back part of the slug, that is in the pre-spore region, thus providing them a potential advantage over the Ancestor strain. However, a sizeable posterior portion of chimeric slugs regularly breaks off at the point of transition between Top-enriched and Ancestor-enriched regions (Fig. 8).

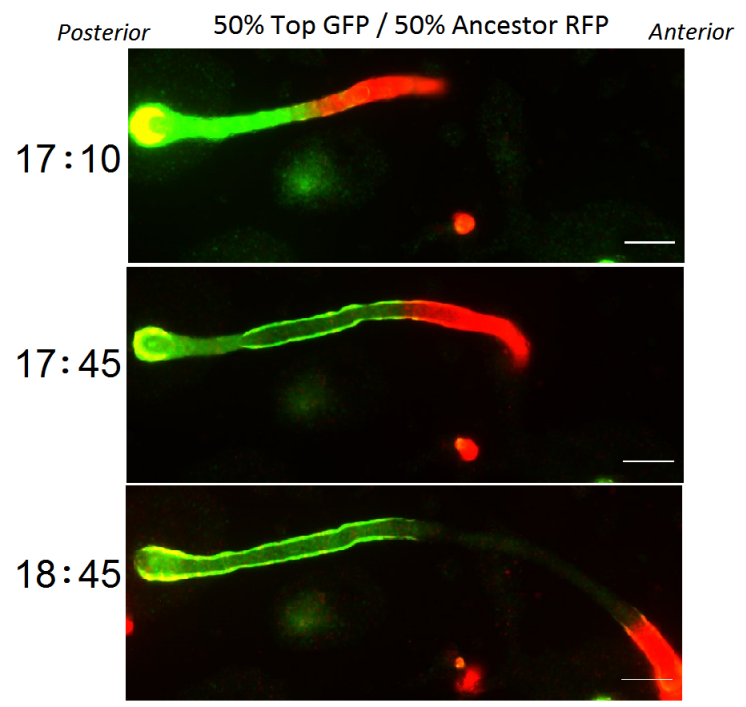

Fig 8. Breaking slug during migration of a Top and Ancestor chimeric slug: the slug regularly breaks off at the point of transition between Top-enriched and Ancestor-enriched regions. The separation typically happens just after the slug emerges from the mound, so that a significant fraction of Top cells remain in proximity of aggregation centers, but they do not develop further. Scale bar $=200 \mu \mathrm{m}$. 
The detached fragments, that lost the traction provided by Ancestor cells, and possibly also the capacity for efficient sorting, do not develop any further. Top cells that remain in the slug end up almost exclusively in the spore mass. At the population level, this is nonetheless insufficient to compensate for the earlier segregation. So, less adhesive cells behave, within single slugs, as extreme cheaters, as the collective functionalities are entirely provided by Ancestor cells. On the other hand, differential adhesion allows Ancestors to 'shrug off' cheaters and protect themselves from their asocial behaviour. It is to be expected that the efficiency of segregation depends on several factors other than differential adhesion, most importantly the fraction of Top cells in the population. When they are in small proportion, indeed, it is possible that Top cells manage to stick to the slug, thus obtaining a disproportionate advantage over the Ancestor. Further investigations of this hypothesis are under way.

The qualitative differences in spore allocation evidenced through Equation 4, and not captured by Equation 2 (summarized in S6 Table: Metrics of reproductive success in chimeras and deviations in competitive spore allocation of each strain compared to their inherent allocation thus correspond to distinct developmental pathways of Top and Bottom cells in chimeras. Such differences in development seem to be related to cell-surface adhesion both directly and indirectly, though cell-cell adhesion. Molecular studies will be needed to disentangle the interplay of these different mechanical forces and cell differentiation during development.

\section{Discussion}

In this work, we explored how selection acting on single-cell properties affects collective behaviour in cellular populations. Understanding how single-cell properties shape adaptations to collective living is key to explore possible evolutionary scenarios leading from unicellular to multicellular life cycles $9,40,41$ In our experimental design, selection on cell-substrate adhesion is applied to the 'social amoeba' D. discoideum at the unicellular stage of its life cycle, thus it does not act directly on social traits. Two evolved strains were obtained, that have higher (Bottom) and lower (Top) substrate adhesion with respect to their common ancestor. Given the relatively small divergence time and mutation rate $\left(2.9 \times 10^{-} 11\right.$ for the nuclear DNA 42$\left.]\right)$, we expect that the three strains retain an almost complete genetic identity. Direct selection of a quantitative cell trait allowed us to factor out the effects of modified adhesion from those of broader genetic differences that occur in natural strains, and, unlike in mutagenized strains, to connect trait difference to their generative selection process. Chimeras containing the derived and the Ancestor strains were used to assess the extent to which social behaviour can evolve as a byproduct of evolution of cell-level physical properties [40,43. We could interpret the resulting patterns of cell sorting based on associated variations in cell-substrate and cell-cell adhesion, and thus access the mechanisms underpinning alternative measures of social performance. In particular, we showed that different metrics provide a different classification of a strain's behaviour.

We focused on cellular adhesion because it has been frequently associated to 'social behaviour' in chimeras, as it can support assortment of cooperative cells. Different forms of cell-cell adhesion are involved in the assortative processes in the course of aggregation and development, and have potential evolutionary bearings. Mechanisms that allow genetically similar cells to recognize one other are often invoked in explaining how kin selection can maintain cooperative behaviour in spite of genetic heterogeneity. For instance, Tigr proteins provide a lock-and-key mechanism for specific recognition of cells that share the same allele at that locus [11,44. Similarly, the csA gene, that encodes a cell adhesion protein anchored in the cell membrane, has been proposed to act as a 'green beard' signal associated to cooperative behaviour [12]. It has nonetheless 
been pointed out that non-specific adhesion can also play a constructive role in the evolution of social behaviour, and could have been determinant in early stages of the evolution of aggregative (as well as clonal) multicellularity 13, 15 17]. Moreover, cell sorting, a central process in morphogenesis, is affected by cell-substrate adhesion through variations in interfacial tension, to which it is coupled through the acto-myosin cortex [45 47]. The possible implication of adhesion to surfaces in the evolution of aggregative multicellularity has not, to our knowledge, been previously explored.

A first form of social behaviour is that manifest in the multicellular cycle of cells of the same genotype. Modifications in cell-substrate adhesion produced by knocking out specific genes was reported to affect aggregation, developmental timing and differentiation [48]. Similar effects of substrate adhesion on developmental patterns were observed in our evolved strains, both in clonal development and in chimeras. Cells of the Bottom strain, more adhesive to the substrate, were particularly enriched in the anterior of chimeric slugs. This is consistent with the previous observation that anterior cells display, upon disaggregation of the slug, stronger substrate adhesion than posterior cells 49, suggesting that cell sorting in the slug is generally influenced, directly or indirectly (for instance, through a correlated change in polar cell adhesion) by cell-substrate adhesion systems. The Top strain, less adhesive to the substrate, differed from the Ancestor in other ways. During aggregation, it displayed patterns similar to those obtained when cell-substrate adhesion was reduced by altering the chemical properties of the substrate [23], and the timing of clonal development was severely altered. In chimeras, Top cells distributed in the slug like strains where adhesion to the substrate was impaired by knocking out the paxB or dimA- genes [30,50].

Spore production in chimeras of strains with different genetic background - typically natural isolates or mutagenized variants - mixed pairwise are the cornerstone of experimental use of Dictyostelium and, more generally, of cooperation in microbes, to test evolutionary theories. Given the ease of counting spores that derive from one or another strain, social behaviour has been extensively associated to the

over-representation ('cheating') or under-representation ('cooperation') of one genotype in the spore pool 29 32]. We have shown that definition of social behaviour based purely on the fraction of spores produced can actually conceal divergent developmental paths. When spore bias is computed as the distance of spore frequency with respect to cell frequency in the mix (eq. 2) both Bottom and Top strains come to be classified as cooperators. However, observation of the developmental cycle revealed fundamental differences in contribution of the evolved strains to multicellular function. Bottom cells appear to 'sacrifice themselves' by forming the upper cup and the basal disk, which are both essential for the formation and the structural stability of the fruiting body. This apparent cooperative behaviour is likely a passive consequence of stronger attachment to the substrate and of increased polar contacts. Top cells, on the other hand, act within the slug as cheaters, as they tend to position themselves in the back of the slug, and later enter the spore mass. This strategy can be fruitful as long as Ancestor cells can compensate for the recalcitrance of a small proportion of Top cells. Further studies are needed to check if, as expected based on our observations, social success is frequency-dependent, and if, along the lineage of the Top strain, strains that evolved only a slight decrease in adhesion produce a spore bias compatible with unconditional cheating. In the conditions we have examined, lack of coordination between strains results in a counter-productive detachment of Top-enriched tails of streams and slugs. In this way, Top cells end up undermining not only the reproductive success of the Ancestor, but their own as well. Recognition that apparent cooperation can come as a side effect of excessive greed is captured by metrics that, in computing the expected composition of the spore pool, also consider the amount of spores produced by each strain on its own (eq. 44. This metric (as that proposed in Buttery et al. 33]) takes into 
account that one strain may produce more spores in the first place, which would give it a head start in the chimera if the decision of a cell to turn into a spore was independent of the social interactions. The second definition seems to better reflect the developmental differences observed in evolved strains. Beside integrating information on the 'ecological' dynamics within a life cycle, such more general metric may also be more relevant to predicting the evolutionary success of strains in contexts where populations are structured in aggregates of variable composition (unlike what typically happens on the scale of lab experiments).

One recognized mechanism acting within a single life cycle to counterbalance selective pressures acting on specific phenotypic traits is pleiotropy. It has been for instance invoked as the reason why mutants with decreased sensitivity to DIF (differentiation-inducing factor), a morphogen controlling the stalk-specific pathway of differentiation, are not positively selected [30. Despite being considered an important feature of systems where cooperation is mediated by public goods [51], the way pleiotropic effects deploy along an evolutionary trajectory is seldom revealed. In our work, we have identified putative pleiotropic effects of changes in cell-surface adhesion on cell-cell adhesion. Even though we do not know what are the molecular basis of such co-variation, we showed that it is sufficient to qualitatively explain the developmental patterns and social behaviour observed in chimeras.

The action of selection on properties of isolated cells has been so far largely overlooked when considering the evolution of social traits, with the notable exception of the role of 'loner' cells 25,52,53. Here, we showed that properties of isolated cells can be important not only in fluctuating environments, where different behavioural patterns are effective in hedging cells' bets in front of unpredictable selective pressures. Through their direct and indirect consequences on the multicellular phase of the life cycle, changes in cellular physical properties can oppose selection on multicellular traits and constrain the possible solutions available to development.

Evolutionary effects similar to those we evidenced could be produced by a number of other selective pressures that, acting outside the multicellular phase of the life cycle, end up affecting the way cells coordinate spatially and temporally to achieve a collective function. Possible future investigation may consider whether cell-intrinsic differences in motility or response to the environment are as effective as adhesion in steering the course of social evolution.

\section{Author contributions}

Conceptualization, Methodology, Formal analysis: S.A; Investigation: S.A and M.F; Writing - Original Draft: S.A and S.D.M.

\section{Acknowledgments}

The authors are very grateful to Clement Nizak for helpful discussions. This work has received support under the program Investissements d'Avenir launched by the French Government and implemented by ANR with the references ANR-10-LABX-54 MEMOLIFE and ANR-10-IDEX-0001-02 PSL* Université Paris, Q-life ANR-17-CONV-6150005, and the project ANR-19-CE45-0002 'ADHeC' PSL research University.

\section{Declaration of Interests}

The authors declare no competing interests. 


\section{References}

1. Ackermann M. A functional perspective on phenotypic heterogeneity in microorganisms. Nature Reviews Microbiology. 2015;13(8):497-508. doi:10.1038/nrmicro3491.

2. Rainey PB, De Monte S. Resolving conflicts during the evolutionary transition to multicellular life. Annual Review of Ecology, Evolution, and Systematics. 2014;45:599-620.

3. Strassmann JE, Zhu Y, Queller DC. Altruism and social cheating in the social amoeba Dictyostelium discoideum. Nature. 2000;408(6815):965-967.

4. Li SI, Purugganan MD. The cooperative amoeba: Dictyostelium as a model for social evolution. Trends in Genetics. 2011;27(2):48-54.

5. Fortunato A, Strassmann J, Santorelli L, Queller D. Co-occurrence in nature of different clones of the social amoeba, Dictyostelium discoideum. Molecular ecology. 2003;12(4):1031-1038.

6. Castillo D, Queller D, Strassmann J. Cell condition, competition, and chimerism in the social amoeba Dictyostelium discoideum. Ethology Ecology \& Evolution. $2011 ; 23(3): 262-273$.

7. Strassmann JE, Queller DC. How social evolution theory impacts our understanding of development in the social amoeba Dictyostelium. Development, growth \& differentiation. 2011;53(4):597-607.

8. Grosberg RK, Strathmann RR. The evolution of multicellularity: a minor major transition? Annu Rev Ecol Evol Syst. 2007;38:621-654.

9. Márquez-Zacarías P, Conlin PL, Tong K, Pentz JT, Ratcliff WC. Why have aggregative multicellular organisms stayed simple? Current Genetics. 2021; p. $1-6$.

10. Forget M, Adiba S, De Monte S. Social conflicts in Dictyostelium discoideum: a matter of scales. 2021;

11. Hirose S, Benabentos R, Ho HI, Kuspa A, Shaulsky G. Self-recognition in social amoebae is mediated by allelic pairs of tiger genes. Science. 2011;333(6041):467-470.

12. Queller DC, Ponte E, Bozzaro S, Strassmann JE. Single-gene greenbeard effects in the social amoeba Dictyostelium discoideum. Science. 2003;299(5603):105-106.

13. Ispolatov I, Ackermann M, Doebeli M. Division of labour and the evolution of multicellularity. Proceedings of the Royal Society B: Biological Sciences. 2012;279(1734):1768-1776.

14. Garcia T, De Monte S. Group formation and the evolution of sociality. Evolution: International Journal of Organic Evolution. 2013;67(1):131-141.

15. Garcia T, Brunnet LG, De Monte S. Differential adhesion between moving particles as a mechanism for the evolution of social groups. PLoS computational biology. 2014;10(2).

16. Garcia T, Doulcier G, De Monte S. The evolution of adhesiveness as a social adaptation. Elife. 2015;4:e08595. 
17. van Gestel J, Wagner A. Cryptic surface-associated multicellularity emerges through cell adhesion and its regulation. PLoS Biology. 2021;19(5):e3001250.

18. Kessin RH. Dictyostelium: evolution, cell biology, and the development of multicellularity. vol. 38. Cambridge University Press; 2001.

19. Raper KB. The dictyostelids. Princeton University Press; 2014.

20. De Laat SW, Van Der Saag PT, Shinitzky M. Microviscosity modulation during the cell cycle of neuroblastoma cells. Proceedings of the National Academy of Sciences. 1977;74(10):4458-4461.

21. Gerisch G. Chapter 11 Univalent Antibody Fragments as Tools for the Analysis of Cell Interactions in Dictyostelium. In: Moscona AA, Monroy A, editors. Immunological Approaches to Embryonic Development and Differentiation Part II. vol. 14 of Current Topics in Developmental Biology. Academic Press; 1980. p. 243 - 270. Available from: http://www.sciencedirect.com/science/article/pii/S0070215308601970.

22. Fujimori T, Nakajima A, Shimada N, Sawai S. Tissue self-organization based on collective cell migration by contact activation of locomotion and chemotaxis. Proceedings of the National Academy of Sciences. 2019;116(10):4291-4296.

23. Wang C, Chowdhury S, Driscoll M, Parent CA, Gupta SK, Losert W. The interplay of cell-cell and cell--substrate adhesion in collective cell migration. Journal of The Royal Society Interface. 2014;11(100):20140684.

24. Rosengarten RD, Santhanam B, Fuller D, Katoh-Kurasawa M, Loomis WF, Zupan B, et al. Leaps and lulls in the developmental transcriptome of Dictyostelium discoideum. BMC genomics. 2015;16(1):1-16.

25. Dubravcic D, Van Baalen M, Nizak C. An evolutionarily significant unicellular strategy in response to starvation in Dictyostelium social amoebae. F1000Research. 2014;(3). doi:10.12688/f1000research.4218.2.

26. Nizak C, Fitzhenry RJ, Kessin RH. Exploitation of other social amoebae by Dictyostelium caveatum. PloS one. 2007;2(2):e212. doi:org/10.1371/journal.pone.0000212.

27. Gerisch G. Differentiation in Dictyostelium. Current topics in developmental biology. 1968; p. 157.

28. Schindelin J, Arganda-Carreras I, Frise E, Kaynig V, Longair M, Pietzsch T, et al. Fiji: an open-source platform for biological-image analysis. Nature methods. $2012 ; 9(7): 676-682$.

29. Fortunato A, Queller DC, Strassmann JE. A linear dominance hierarchy among clones in chimeras of the social amoeba Dictyostelium discoideum. Journal of evolutionary biology. 2003;16(3):438-445.

30. Foster KR, Shaulsky G, Strassmann JE, Queller DC, Thompson CR. Pleiotropy as a mechanism to stabilize cooperation. Nature. 2004;431(7009):693-696.

31. Santorelli LA, Thompson CR, Villegas E, Svetz J, Dinh C, Parikh A, et al. Facultative cheater mutants reveal the genetic complexity of cooperation in social amoebae. Nature. 2008;451(7182):1107-1110. 
32. Santorelli LA, Kuspa A, Shaulsky G, Queller DC, Strassmann JE. A new social gene in Dictyostelium discoideum, chtB. BMC evolutionary biology. 2013;13(1):1-10.

33. Buttery NJ, Rozen DE, Wolf JB, Thompson CR. Quantification of social behavior in D. discoideum reveals complex fixed and facultative strategies. Current Biology. 2009;19(16):1373-1377.

34. Rubin J, Robertson A. The tip of the Dictyostelium discoideum pseudoplasmodium as an organizer. Journal of embryology and experimental morphology. 1975;33(1):227-241.

35. Sriskanthadevan S, Zhu Y, Manoharan K, Yang C, Siu CH. The cell adhesion molecule DdCAD-1 regulates morphogenesis through differential spatiotemporal expression in Dictyostelium discoideum. Development. 2011;138(12):2487. doi:10.1242/dev.060129.

36. Hou L. The role of gp150 and analysis of the relationship between adhesion molecules during Dictyostelium discoideum development. Dong wu xue bao[Acta zoologica Sinica]. 2004;50(1):75-82.

37. Fontana DR. Two distinct adhesion systems are responsible for EDTA-sensitive adhesion in Dictyostelium discoideum. Differentiation. 1993;53(3):139-147. doi:org/10.1111/j.1432-0436.1993.tb00702.x.

38. Desbarats L, Brar SK, Siu CH. Involvement of cell-cell adhesion in the expression of the cell cohesion molecule gp80 in Dictyostelium discoideum. Journal of Cell Science. 1994;107(6):1705.

39. Roisin-Bouffay C, Jang W, Caprette DR, Gomer RH. A precise group size in Dictyostelium is generated by a cell-counting factor modulating cell-cell adhesion. Molecular cell. 2000;6(4):953-959.

40. Arias Del Angel JA, Nanjundiah V, Benítez M, Newman SA. Interplay of mesoscale physics and agent-like behaviors in the parallel evolution of aggregative multicellularity. EvoDevo. 2020;11(1).

41. Pentz JT, Márquez-Zacarías P, Bozdag GO, Burnetti A, Yunker PJ, Libby E, et al. Ecological advantages and evolutionary limitations of aggregative multicellular development. Current Biology. 2020;30(21):4155-4164.

42. Saxer G, Havlak P, Fox SA, Quance MA, Gupta S, Fofanov Y, et al. Whole genome sequencing of mutation accumulation lines reveals a low mutation rate in the social amoeba Dictyostelium discoideum. PloS one. 2012;7(10):e46759.

43. Del Angel JAA, Nanjundiah V, Benítez M, Newman SA. Interplay of mesoscale physics and agent-like behaviors in the parallel evolution of aggregative multicellularity. EvoDevo. 2020;11(1):1-18.

44. Gruenheit N, Parkinson K, Brimson CA, Kuwana S, Johnson EJ, Nagayama K, et al. Cell cycle heterogeneity can generate robust cell type proportioning. Developmental cell. 2018;47(4):494-508. e4.

45. Steinberg MS. Adhesion-guided multicellular assembly: a commentary upon the postulates, real and imagined, of the differential adhesion hypothesis, with special attention to computer simulations of cell sorting. Journal of Theoretical Biology. 1975;55(2):431 - 443. doi:https://doi.org/10.1016/S0022-5193(75)80091-9. 
46. Brodland GW, Chen HH. The mechanics of heterotypic cell aggregates: insights from computer simulations. J Biomech Eng. 2000;122(4):402-407.

47. Brodland GW. The differential interfacial tension hypothesis (DITH): a comprehensive theory for the self-rearrangement of embryonic cells and tissues. J Biomech Eng. 2002;124(2):188-197.

48. Plak K, Pots H, Van Haastert PJ, Kortholt A. Direct Interaction between TalinB and Rap1 is necessary for adhesion of Dictyostelium cells. BMC cell biology. 2016;17(1):1.

49. YABUNO K. Changes in cellular adhesiveness during the development of the slime mold Dictyostelium discoideum. Development, growth \& differentiation. 1971;13(3):181-190.

50. Bukahrova T, Weijer G, Bosgraaf L, Dormann D, van Haastert PJ, Weijer CJ. Paxillin is required for cell-substrate adhesion, cell sorting and slug migration during Dictyostelium development. Journal of Cell Science. 2005;118(18):4295. doi: $10.1242 /$ jcs.02557.

51. Dandekar AA, Chugani S, Greenberg EP. Bacterial quorum sensing and metabolic incentives to cooperate. Science. 2012;338(6104):264-266.

52. Rossine FW, Martinez-Garcia R, Sgro AE, Gregor T, Tarnita CE. Eco-evolutionary significance of "loners". PLoS biology. 2020;18(3):e3000642.

53. Miele L, De Monte S. Aggregative cycles evolve as a solution to conflicts in social investment. PLOS Computational Biology. 2021;17(1):e1008617. 


\section{Supporting information}

S1 Fig. Number of generations of Top, Ancestor and Bottom.

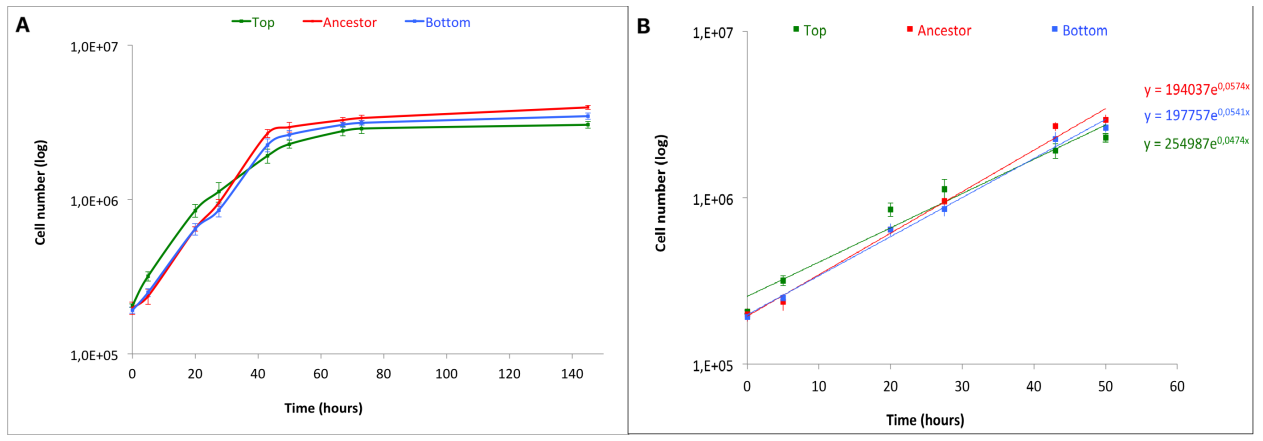

The doubling time $D T$ was calculated at the end of the evolution experiment for Top and Bottom strains by estimating the exponential growth rate. The growth curve (panel A, number of cells measures with the hemocytometer) indicates that cells grow approximately exponentially for 50 hours, before saturation of the population size. This results in linear growth in a semi-logarithmic plot (panel B), the slope $a$ of which was computed by linear regression $(a=0.0474 ; 0.0541$ and 0.0574 for Top, Bottom and Ancestor respectively). From this, we estimate the doubling time $D T=\frac{\ln 2}{a}$ ) as 14.6 hours, 12.8 hours and 12.1 hours for Top, Bottom and Ancestor respectively. So, selection had an effect on growth rate, but changes were likely contained by the experimental design, that alternated selective passaging with undisturbed growth.

Overall, the difference in number of generations was modest. The evolution experiment, indeed, was performed during $D=52$ days, corresponding to a number of generations $\left(G=\frac{D}{D T}\right)$ approximately equal to 85 and 97 for Top and Bottom, respectively. (colour code as in Fig. 2).

S2 Fig. Cell-substrate adhesion during growth, at starvation and during streaming.

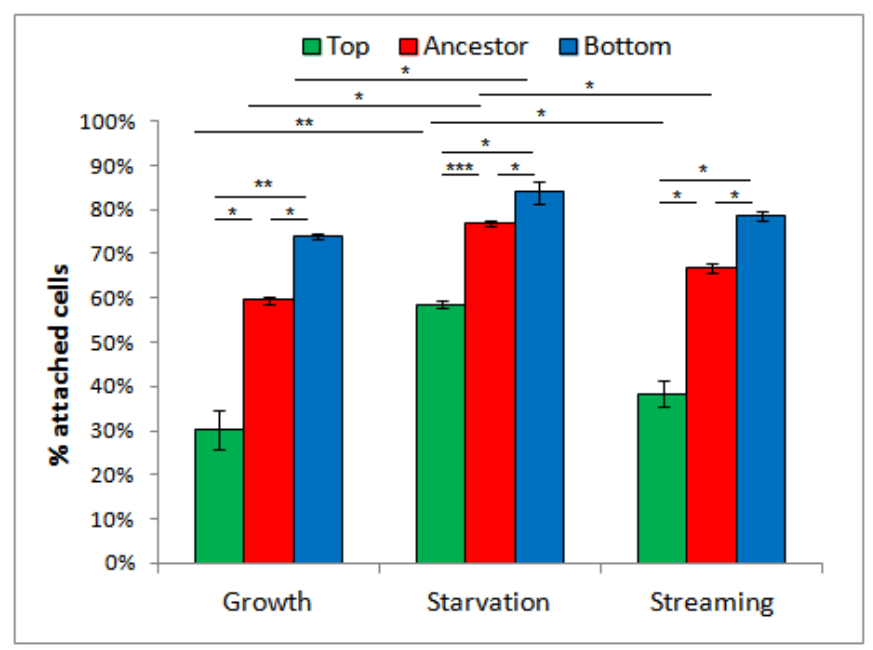

Although cell-substrate adhesion changes in time, qualitative differences among strains are maintained. (colour code as in Fig. $2 .{ }^{*} \mathrm{p}<0.05,{ }^{* *} \mathrm{p}<0.001,{ }^{* * *} \mathrm{p}<0.0001$ ). 
S3 Movie: Ancestor, Top and Bottom alone. Phase contrast images: Developmental life cycle of Ancestor (on the left), Top (on the middle) and Bottom (on the right). Beginning of the different stages of the multicellular developmental cycle (summarized in Fig 2) are indicated.

S4 Fig. Aggregate mean area $\left(\mathrm{mm}^{2}\right)$ and number.

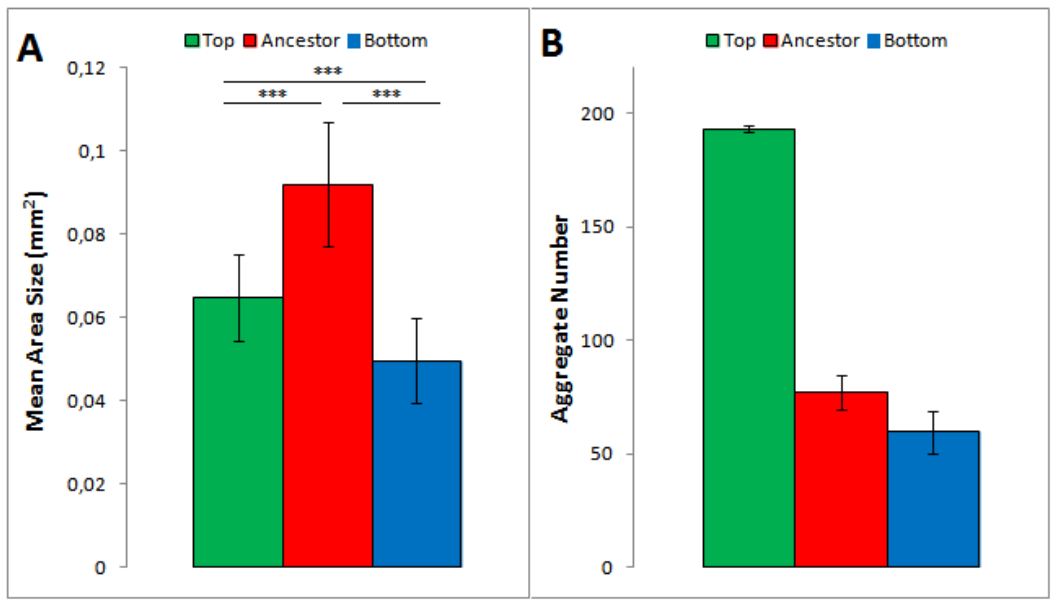

A: Mean aggregate area (averaged over two independent replicates). Aggregates were smaller for Top and Bottom compared to Ancestor. B: Aggregate number for Top, Ancestor and Bottom. Number of aggregates was higher for Top compared to Ancestor and Bottom. $(* * * \mathrm{p}<0.0001)$. (colour code as in Fig. $\left.2{ }^{* * *} \mathrm{p}<0.0001\right)$.

S5 Fig. Spore bias and spore formation efficiency in chimeras.

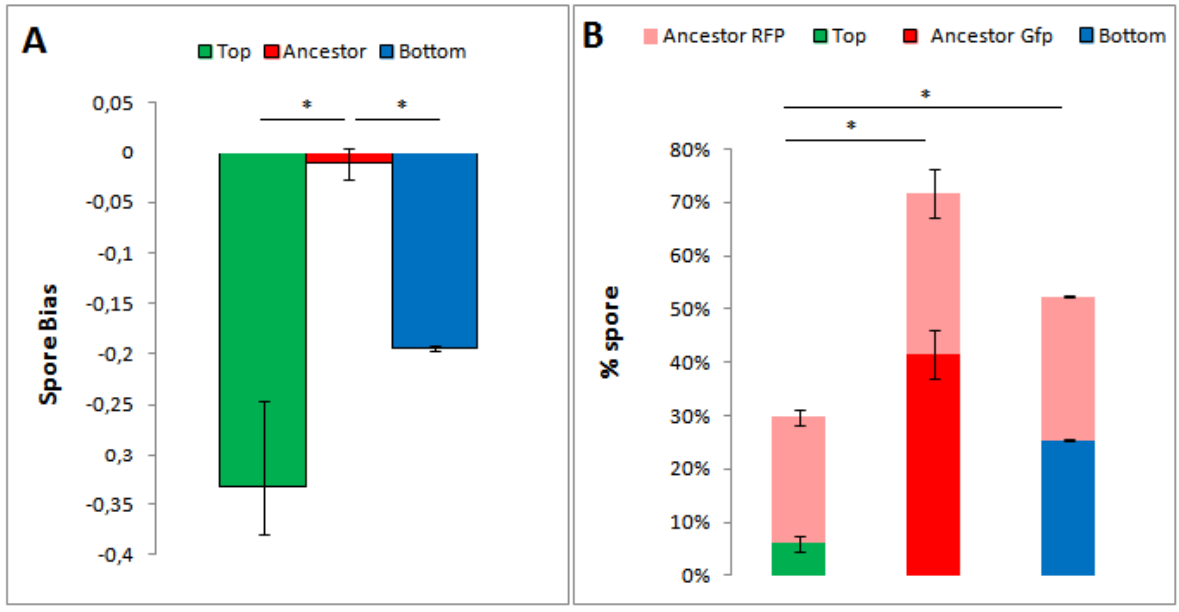

Chimeras were formed by mixing an RFP-tagged Ancestor with GFP-tagged

Ancestor, Top and Bottom. A: Bias in spore allocation of the focal strain relative to its initial proportion of cells in the mix (as estimated from Equation 2. Top and Bottom contribute less than the Ancestor to the spore pool (error bars are stardard deviations over 3 replicates, colour code as in Fig. 22. B: Number of spores of the two co-aggregating strains (the RFP-tagged Ancestor strain is indicated in pink) relative to the number of cells of that strain in the mix: spore formation efficiency is significantly reduced when the Ancestor strain is mixed with both Top and Bottom $\left({ }^{*} \mathrm{p}<0.05\right)$. 
S6 Table: Metrics of reproductive success in chimeras and deviations in competitive spore allocation of each strain compared to their inherent allocation. Spore allocation was considered in three different chimeras (TA: chimeras Top-Ancestor; AA: chimeras Ancestor GFP-Ancestor RFP; BA: chimeras Bottom-Ancestor) in triplicate measures. $S F E_{i}$ Spore Formation Efficiency of the strain $i$ ) (Equation 1); $p_{i}$ cell proportion of the strain $i$ plated; $P_{i}$ spore proportion of the strain $i$ after development; $\tilde{P}_{i}^{j}$ expected spore proportion of the strain $i$ when with the strain $j$ (Equation 3); $d_{i}^{j}$ metric of social performance (Equation 4) : positive values indicate 'cheating', negative values 'cooperation'.

\begin{tabular}{cccccc} 
& $S F E_{i}$ & $p_{i}$ & $P_{i}$ & $\tilde{P}_{i}^{j}$ & $d_{i}^{j}$ \\
\hline Top & 0.10 & \multirow{2}{*}{0.54} & 0.20 & 0.130 & 0.07 \\
(TA) & \pm 0.01 & & \pm 0.09 & \pm 0.003 & \pm 0.09 \\
\hline Ancestor & 0.79 & \multirow{2}{*}{0.50} & 0.49 & 0.51 & -0.02 \\
(AA) & \pm 0.09 & & \pm 0.05 & \pm 0.09 & \pm 0.1 \\
\hline Bottom & 0.49 & \multirow{2}{*}{0.64} & 0.254 & 0.53 & -0.27 \\
(BA) & \pm 0.02 & & \pm 0.003 & \pm 0.02 & \pm 0.02 \\
\hline
\end{tabular}

S7 Movie: multicellular developmental cycle in a chimera between (lower left) and RFP (lower right) channels, and merged (upper right) during the multicellular life cycle.

S8 Movie: multicellular developmental cycle in a chimera between Top and Ancestor. Time-lapse movies in phase contrast (upper left), GFP (lower left) and RFP (lower right) channels, and merged (upper right) during the multicellular life cycle.

S9 Movie: multicellular developmental cycle in a chimera between Bottom and Ancestor. Time-lapse movies in phase contrast (upper left), GFP (lower left) and RFP (lower right) channels, and merged (upper right) during the multicellular life cycle. 